\title{
Method and results of comparing multilevel spectral reflectance measurements of various Earth surfaces
}

\author{
L.V. Katkovsky ${ }^{1}$, B. I. Beliaev ${ }^{1}$, M. Yu. Beliaev², A. M. Esakov², D. A. Ivanov ${ }^{1}$, \\ A. O. Martinov ${ }^{1}$, V. O. Siliuk ${ }^{1}$, E. E. Sarmin ${ }^{2}$ \\ ${ }^{1}$ A. N. Sevchenko Institute of Applied Physical Problems of Belarusian State \\ University, Minsk, 220045, Republic of Belarus \\ ${ }^{2}$ S. P. Korolev Rocket and Space Public Corporation Energia, Korolev, 141070, Russia \\ E-mail: remsens@mail.ru
}

\begin{abstract}
The paper describes a method and results of comparison of quasi-synchronous space, aviation and ground-based measurements of the reflection spectra of various underlying surfaces. The purpose of such measurements is to obtain data for vicarious calibrations of satellite spectral sensors, verification of space and aviation data, as well as post-processing of this data. Measurements from the Photospectral system (PhSS) from the International space station (ISS) and images of some satellites (Landsat 8, Sentinel-2, etc.) were used as space information. As objects of research were the test site in the Gomel region (Republic of Belarus) and the volcanoes of the Kamchatka Peninsula. Spectral radiance and spectral reflectance that were measured were compared in this study. For aircraft data, the measured reflection spectra were recalculated to the top of the atmosphere (TOA) into the satellite measurements conditions, and the atmospheric correction of the aircraft spectra was carried out to obtain the reflectance at the underlying surface. Satisfactory agreement between ground-based, aviation spectra and spaceborne measurements suggests that the ground and aviation-based spectra can be used to search for the target objects (adaptive filtering) during post-processing of both hyperspectral and multispectral space images of various spatial resolutions.
\end{abstract}

Accepted: 15.09 .2020

DOI: 10.21046/2070-7401-2020-17-6-30-36

\section{Introduction}

The Aerospace Research Department of A. N. Sevchenko Institute of Applied Physical Problems BSU is working on the development of methods for calibration of airborne and spaceborne spectral systems, as well as the design of devices for such multilevel measurements and equipping satellite test sites.

The calibration procedure for satellite devices is carried out to check the stability of the characteristics of the satellite sensors. For this, test sites should be sufficiently long, homogeneous, and also equipped with high-precision spectral equipment, which allows getting the spectra of the studied underlying surface from the ground or from an aircraft carrier. For ground-base and aircraft measurements, the photospectroradiometer FSR was developed. FSR has a high spectral resolution (2-3 nm) in the wavelength range 400-900 $\mathrm{nm}$ [1]. This device includes an image registration system (based on a smartphone) and spectra registration system based on spectroradiometer developed by the Aerospace Research Department. Both systems combined into a single module. FSR also provides spatial reference of spectrometry areas to images, as well as spectra and images to GLONASS/GPS navigation data (time, geographical coordinates), recording it to memory and viewing data. The measurement errors of the absolute radiance data of the underlying surfaces are about $5 \%$. 


\section{ISS onboard measurements}

Within the "Uragan" space experiment, the following scientific equipment is operating on board the ISS: since 2010 - PhotoSpectral System (PhSS) [2] and since 2014 - Video Spectral System (VSS) [3]. During its operation on board the ISS, the PhSS acquired images and spectra of a large number of various Earth's underlying surfaces. This data has high spectral resolutions, RGB images and georeferencing allows to determine the type of underlying surfaces to which the measured spectra correspond. For processing PhSS data were developed specific methods, including atmospheric correction. By the results of PhSS data processing a spectral reflectance library of a large number of various underlying surfaces, which are often unavailable for ground-based measurements, was created. The library can be used to prepare initial data for space experiments on remote sensing and searching for target objects.

Before the creation of the spectral reflectance library of PhSS measurements, some part of the PhSS spectra were validated using ground-based measurements obtained in the RadCalNet project, which is an initiative of the Working Group on Calibration and Validation of the Committee on Earth Observation Satellite (https://www.radcalnet.org/\#!/). One of the four test sites of the RadCalNet network is located in Namib desert (Namibia) in near the village of Gobabab and is codenamed GONA. This test site was chosen for validation with $\mathrm{PhSS}$ data, since there is a large number of spectra of various deserts obtained by the PhSS. For validation, we selected a number of sand radiance spectra, acquired by the PhSS on June 21, 2011, August 30, 2011, and March 30, 2012. From these radiance spectra, the reflectances were calculated using the developed method of atmospheric correction [4]. To validate the PhSS data on RadCalNet, several measurements were taken from the GONA site at different years. The spectral reflectance calculated from the PhSS data, as well as measured at the GONA test site, are shown in figure 1. There is a good agreement of the spectral reflectance obtained from the PhSS data and measured at the GONA test site at different years.

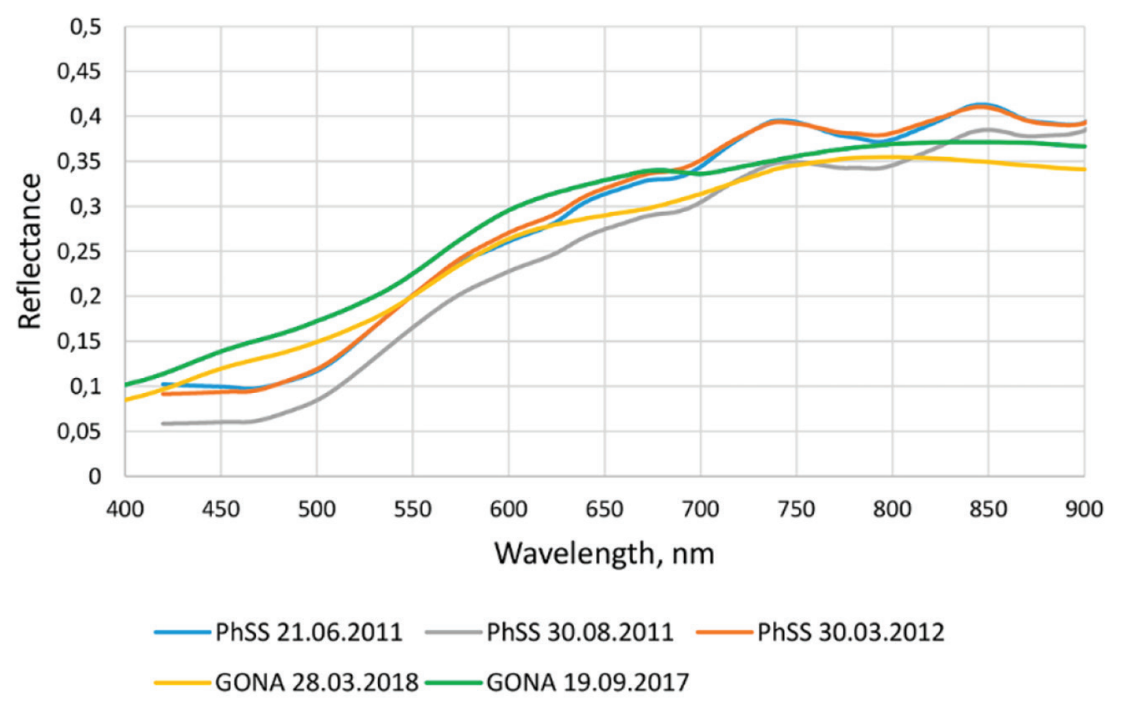

Figure 1. Spectral reflectance calculated by atmospheric correction of PSS data from ISS and measured at the GONA test site (Namibia).

The mean coefficient of determination $R^{2}$ for PhSS measurements and the sand spectrum measured on GONA site acquired on 19.09.2017 and on 28.03.2018 was 0.816 and 0.863 , respectively. This result implies the reliability of the spectral reflectance data, calculated from the PhSS data, the longterm stability of the reflection spectra of sand, and the possibility of using the reflectance library of various underlying surfaces recorded from the spaceborne imagery data.

\section{Multilevel spectral measurements}

In order to test the validation method of multilevel measurements during experiments with the implementation of quasi-synchronous shooting with the PhSS, VSS equipment on-board the ISS, a series of measurements in the following sites were performed: 
1. Zyabrovka test site (Gomel region, Belarus), on August-September of 2018. Type of underlying surface: concrete runway of the airfield, $3 \mathrm{~km}$ long and $80 \mathrm{~m}$ wide; vegetation near the airfield (a mixture of meadow vegetation with a predominance of acute sedge (Carex acuta L.); spoil tips of the Gomel Chemical Plant: heaps of unrefined phosphogypsum of bright white or gray color with an area of about $1.5 \mathrm{~km}^{2}$. The measurements were carried out at three levels: groundbased, airborne $(\sim 1 \mathrm{~km})$ and spaceborne. Since it was not possible to obtain quasi-synchronous measurements with on-board the ISS sensors, the ground-based spectrometry data for the described objects were validated with the images of this territory of the Sentinel-2B multispectral sensor. The satellite image areas that cover the specified ground-based measurement area are shown on figure 2. The marks indicate the points of the ground-based measurements. Satellite and ground-based measurements were carried out on the same day on September 20, 2018.
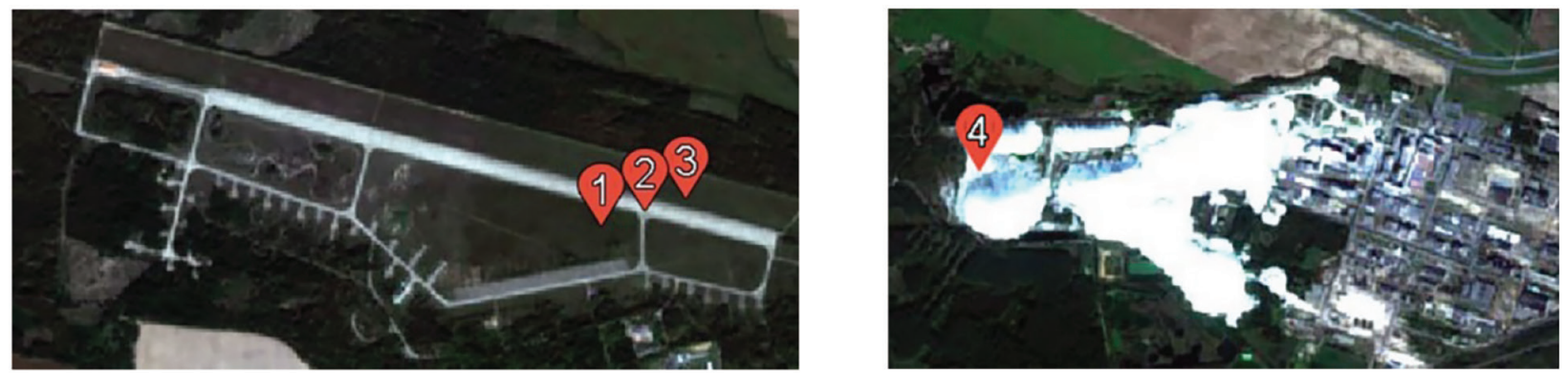

Figure 2. Sentinel-2 image areas that cover Zyabrovka test site.
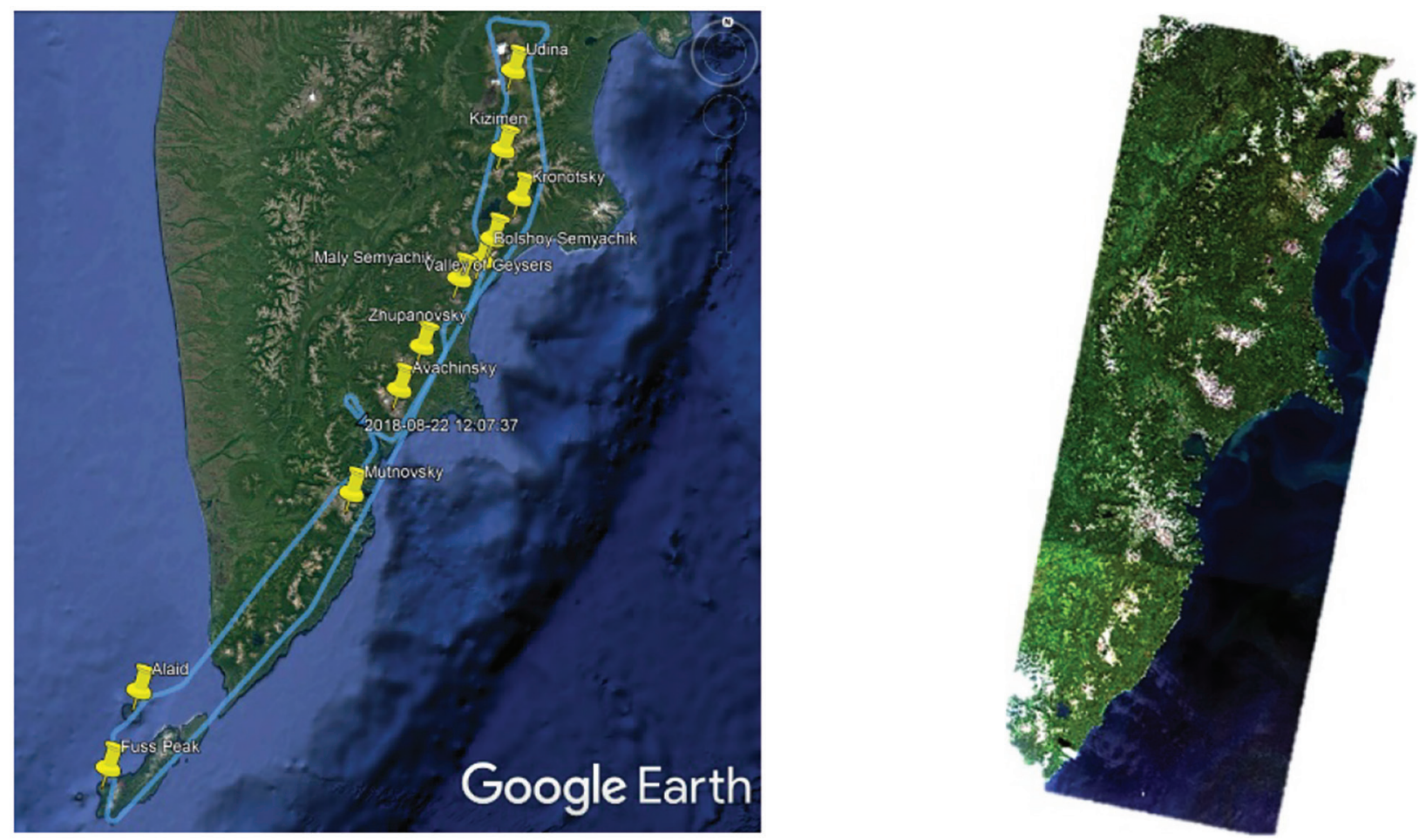

Figure 3. Left: overflight path (blue line) and objects whose spectra were obtained (yellow labels); right: Landsat- 8 data covering the area of the flight.

2. Russia's Far East volcanoes, August 2018. Type of underlying surface: a number of volcanoes on the Kamchatka Peninsula, Geyser's Valley, volcanoes of the Kuril Islands. Due to the difficult to reach of these objects for ground-based measurements, airborne $(\sim 10 \mathrm{~km})$ and spaceborne measurements of this region were performed. To carry out the validation, satellite images of the study area, acquired by the Landsat 8 sensor, were used. Figure 3 shows the tracking data and measured objects, as well as Landsat 8 images covering the overflight area. The Landsat 8 data 
were obtained on the same day when the in-flight airborne measurements were acquired, on August 22, 2018 with a Sun's elevation angle of about $46^{\circ}$ (the elevation angle during aircraft measurements ranged from 45.4 to $46.7^{\circ}$ ). The volcanoes of the Kuril Islands were not captured by the Kamchatka volcanos's series of measurements by a satellite sensor. The image covering the Udina volcano is covered by continuous clouds, therefore, the validation for these objects was not carried out. An example of data acquired by the satellite sensor and the camera of the FSR smartphone is shown in figure 4.
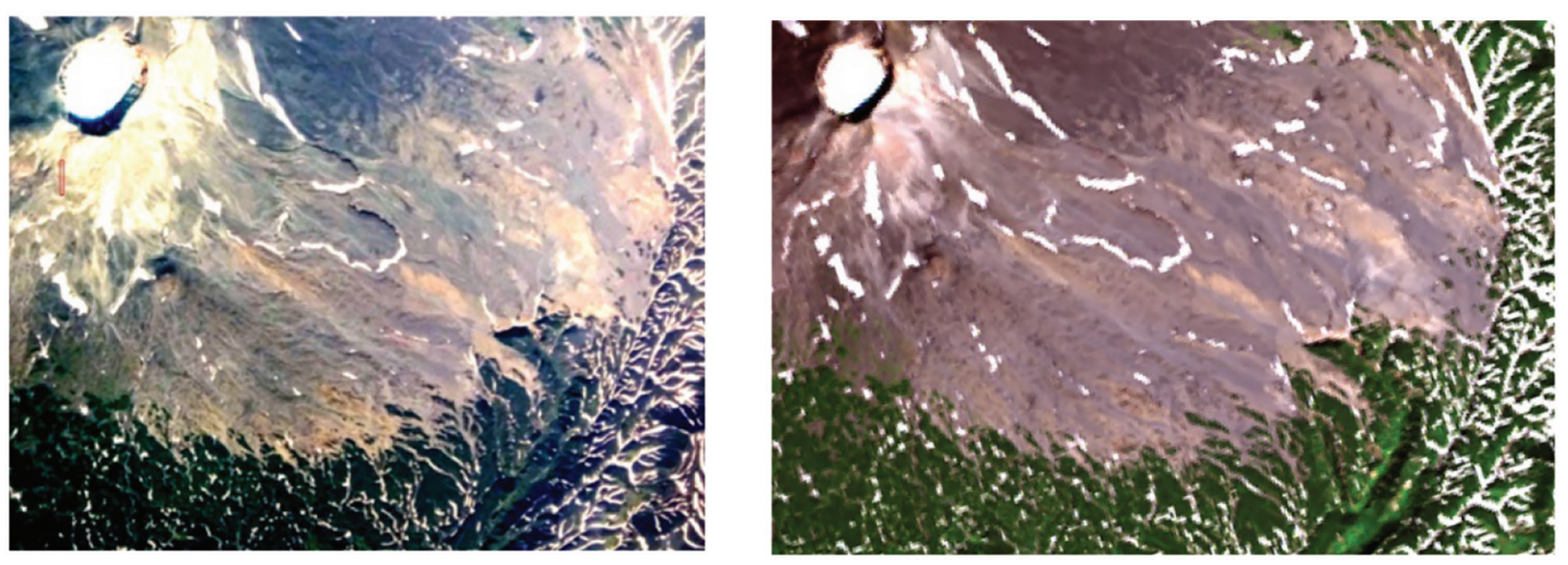

Figure 4. Image of the Maly Semyachik volcano, recorded by: left - an FSR smartphone; right - Landsat 8 sensor.

The algorithm for processing and validating airborne and satellite measurements for the Far East volcanoes case is shown in figure 5.

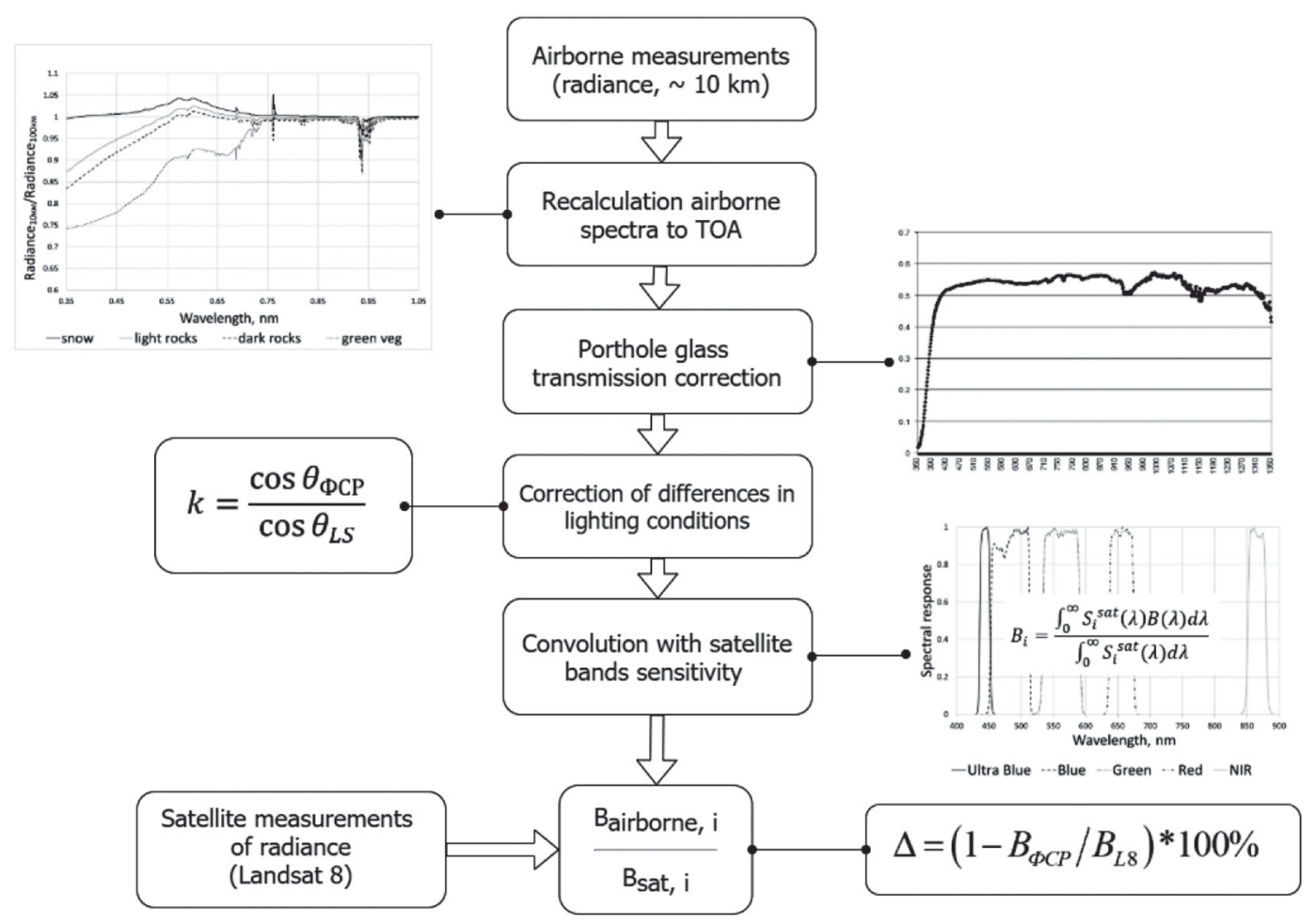

Figure 5. Algorithm of comparison of airborne and satellite measurements.

It should be noted that in calculating the $\Delta$ error, an averaged radiance measured by the satellite sensor of area $12 \times 2$ pixels was taken, which approximately corresponds to the spectrometric area by the FSR from an altitude of $10 \mathrm{~km}$ and a field of view of $2.2 \times 0.25^{\circ}$. 
Moreover, the validation using the surface albedo values was performed. Since Landsat- 8 reflectance data (processed with Landsat Surface Reflectance Code) are available, we obtained the underlying surface albedo spectra by performing atmospheric correction of the measured spectra using a developed technique [4]. This atmospheric correction method includes an optical-physical model of the atmosphere and is based on the use of analytical equations for the spectral radiance of outgoing radiation at the TOA (conventionally $100 \mathrm{~km}$ ) or at any other altitude in the range of $0-100 \mathrm{~km} \mathrm{[5].}$

Validation of ground-based and satellite measurements of the Zyabrovka test site was carried out according to the method, the algorithm of which is shown in figure 6. At comparing satellite and ground-based measurements, the error value $\Delta$ was also calculated using the Equation from figure 5.

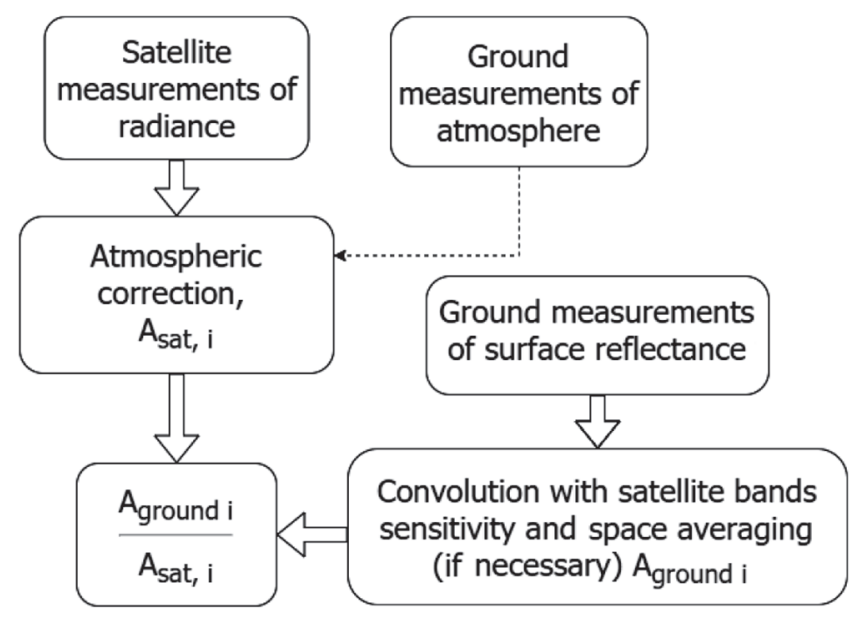

Figure 6. Algorithm of comparison of groundbased and satellite measurements.

\section{Validation results of ground-based and satellite measurements}

The results of validation of ground-based and satellite measurements of the Zyabrovka test site obtained by the FSR and the Sentinel-2 sensor are presented in table 1 .

Table 1. Values of $\Delta$ in case of ground-based and satellite data validation.

\begin{tabular}{lcccc}
\hline Surface & \multicolumn{4}{c}{$\Delta, \%$} \\
\cline { 2 - 5 } & $\mathrm{B}$ & $\mathrm{G}$ & $\mathrm{R}$ & $\mathrm{NIR}$ \\
\hline Point 1, grass & 26.89 & 28.06 & 12.02 & 7.28 \\
Point 2, runway & 1.45 & 1.81 & 1.27 & 7.49 \\
Point 3, vegetation & 2.96 & 1.4 & 2.52 & 1.71 \\
Point 4, spoil tips & 5.69 & 1.85 & 1.35 & 13.86 \\
\hline
\end{tabular}
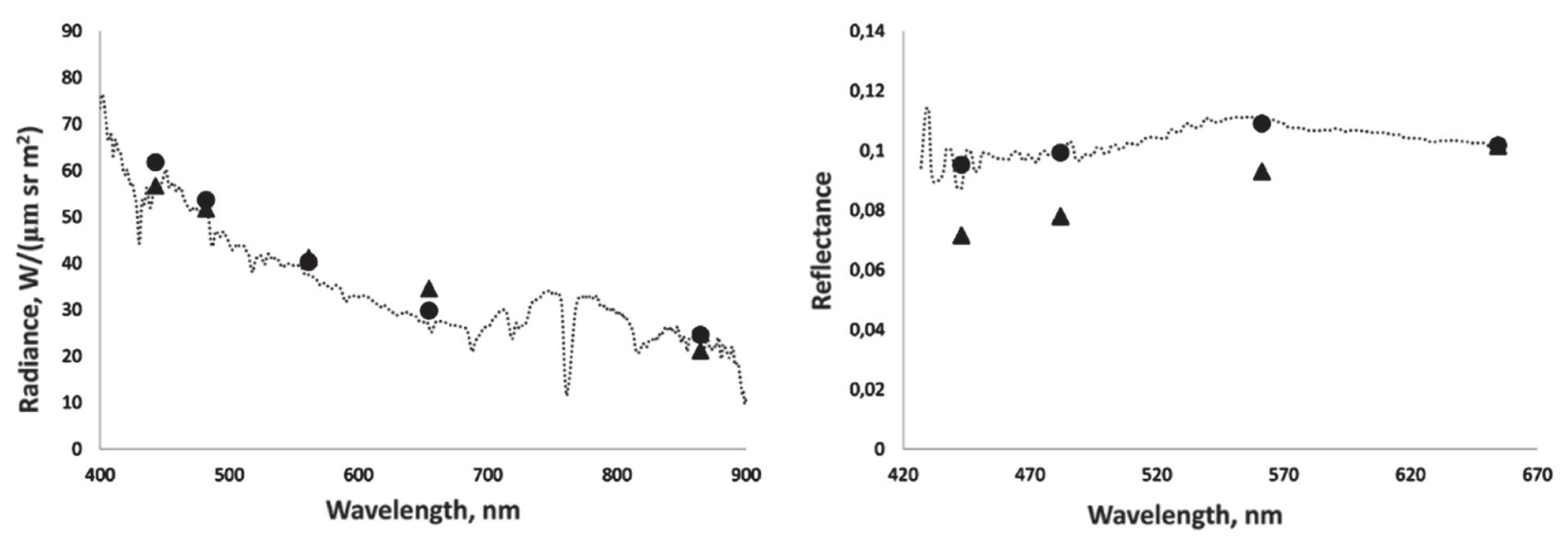

Figure 7. Results of validation of FSR and Lыandsat-8 data for Maly Semyachik volcano ( $\mathbf{\Delta}$ - Landsat-8 data; - - FSR data (convolved with Landsat-8 bands sensitivity); dotted line - initial radiance/reflectance registered by FSR: left - TOA radiance; right - reflectance. 
The results of validation of airborne and satellite measurements of objects on the Kamchatka Peninsula (volcanoes mostly) are presented in table 2. Radiance and reflectance calculated by measurements of Maly Semyachik volcano (Figure 4) by two sensors are shown on figure 7.

Table 2. Values of $\Delta$ in case of airborne and satellite data validation.

\begin{tabular}{lrrrrrrrrr}
\hline Object & \multicolumn{4}{c}{ Radiance } & \multicolumn{5}{c}{ Reflectance } \\
\cline { 2 - 10 } & \multicolumn{1}{c}{ UB } & \multicolumn{1}{c}{ B } & \multicolumn{1}{c}{ G } & \multicolumn{1}{c}{ R } & NIR & UB & \multicolumn{1}{c}{ B } & \multicolumn{1}{c}{ G } & \multicolumn{1}{c}{ R } \\
\hline Valley of Geysers & -1.73 & 3.2 & 11.0 & 24.12 & 2.2 & 4.67 & 2.89 & 1.34 & 21.13 \\
Bolshoy Semyachik & 8.97 & 10.63 & 10.09 & 11.44 & 8.57 & -88.87 & -71.82 & -35.82 & -15.78 \\
Maly Semyachik & -8.86 & -3.62 & 2.17 & 13.95 & -15.85 & -32.99 & -57.29 & -17.25 & -0.12 \\
Zhupanovsky & 13.27 & 18.22 & 22.22 & 24.8 & 17.8 & 29.28 & 24.58 & 29.46 & 35.33 \\
Avachinsky & -9.77 & 0.59 & 13.9 & 25.05 & 57.17 & 8.98 & 11.31 & 15.7 & 19.06 \\
Mutnovsky & 14.81 & 20.05 & 24.64 & 29.86 & 41.75 & -2.1 & 6.19 & 15.94 & 22.58 \\
$\langle\Delta\rangle$ & 9.17 & 8.68 & 14.0 & 22.9 & 22.68 & 27.78 & 27.17 & 16.94 & 18.59 \\
\hline
\end{tabular}

\section{Discussion}

There are the following reasons for the differences in measurements of spectral radiance and albedo data by the two sensors:

- FSR instrumental error, $~ 5 \%$ [6].

- Landsat 8 and Sentinel-2 instrumental error, $~ 5 \%$ [7].

- Spatial coregistration errors and spatial resolution coordination.

- Narrow field of view of the FSR spectrometer (at ground-based measurements, the heterogeneity of the vegetation has a great influence).

- Unaccounted differences in the conditions of the spectra measuring (for example, the shooting angle). In our assumption all measurements were made at nadir.

- Methodological errors associated with the recalculation of airborne spectra to the TOA, and atmospheric correction errors, both of these errors may in some cases (depending on the underlying surface) reach up to $10 \%$.

The most applicable surfaces that can be used as the test sites during satellite sensors vicarious calibration are concrete surfaces (runways) and waste heaps of the Gomel Chemical Plant. The surface of these objects is quite homogeneous and stable. In addition, the concrete runway is a perfectly flat surface. In the area of waste heaps, is possible to find areas with a flat surface as well.

As a result of the validation of airborne and satellite measurements, it can be concluded that the data obtained by the FSR are reliable. The differences that were obtained in the effective spectral radiance of FSR and the Landsat-8, Sentinel-2 satellites sensors over the same underlying surfaces with quasi-synchronous measurements are within the total uncertainties determined by the accuracy of the absolute energy calibrations of the sensors (about 5-10\%), and the rest of the errors (in some cases) can be explained by the factors mentioned above.

\section{References}

[1] Stanchik V., Khomitsevich A., Photospectroradiometer for field measurements of reflection spectra of objects, Applied Problems of Optics, Informatics, Radiophysics and Condensed Matter Physics, Proc., 2017, pp. $145-147$.

[2] Belyaev B.I., Belyaev M.Yu., Desinov L.V., Rogovets A.V., Ryazantsev V.V., Sarmin E. E., Sosenko V.A., Flight testing of research equipment "Photospectral system" onboard ISS RS, Space Engineering and Technologies, 2014, Vol. 1(4), pp. 22-28.

[3] Beljaev B., Beljaev M., Sarmin E., Gusev V., Desinov L., Ivanov V., Krot Y., Martinov A., Rjazancev V., Sosenko V., Design and flight tests of science hardware video-spectral system on board the Russian segment of the ISS, Space Engineering and Technologies, 2016, Vol. 2(13), pp. 70-79.

[4] Katkovsky L., Martinov A., Siliuk V., Ivanov D., Kokhanovsky A., Fast Atmospheric Correction Method for Hyperspectral Data, Remote Sensing, 2018, Vol. 10(11), 1698, 18 p., available at: https://doi. org $/ 10.3390 /$ rs 10111698 . 
[5] Katkovsky L., Martenov A., Siliuk V., Ivanov D., SHARC method for fast atmospheric correction of hyperspectral data, Remote Sensing Clouds and Atmosphere XXIII, Proc. SPIE, 2018, Vol. 10786, 1078609, 13 p. DOI: $10.1117 / 12.2323455$.

[6] Katkovskii L., Belyaev B., Sosenko V., Ablameiko S., Hardwaresoftware complex "Calibration" for groundbased spectrometry of the underlying surface and atmosphere, $7^{\text {th }}$ Belarusian Space Congress, Proc., 2017, Vol. 2, pp. 36-40.

[7] Landsat-8 (L8) Data Users Handbook, Department of the Interior U. S. Geological Survey, 2018,144 p. 